\title{
VARIABILIDAD DEL ENSAYO ECOTOXICOLÓGICO CON Chironomus calligraphus Goeldi (DIPTERA: CHIRONOMIDAE) PARA EVALUAR CADMIO, MERCURIO Y PLOMO
} José Alberto Iannacone Oliver ${ }^{1}$, Neil Salazar Capcha ${ }^{1}$ y Lorena Alvariño Flores ${ }^{1}$

\section{Resumen}

En la evaluación del riesgo ambiental (ERA) de los ambientes acuáticos contaminados por relaves mineros en el Perú se requiere del empleo de organismos biológicos estandarizados como la larva del insecto Chironomus calligraphus Goeldi. Los organismos biológicos para ser usados como herramientas ecotoxicológicas requieren ser sencillos, prácticos, sensibles y repetibles. Por lo que el objetivo de este trabajo fue evaluar el $\mathrm{Cd}^{+2}, \mathrm{Hg}^{2+}$ y $\mathrm{Pb}^{2+}$ en forma de cloruro de cadmio $\left(\mathrm{CdCl}_{2}\right)$, cloruro de mercurio $\left(\mathrm{HgCl}_{2}\right)$ y nitrato de plomo $\left(\mathrm{Pb}_{3}(\mathrm{NO})_{2}\right)$ respectivamente, sobre la mortalidad de las larvas de primer estadio del díptero C. calligraphus a $48 \mathrm{~h}$ de exposición y así determinar su variabilidad. La especie fue aclimatada y criada en condiciones estandarizadas de laboratorio. Se realizaron seis bioensayos de toxicidad aguda estáticos para cada metal, en cada bioensayo se siguió un diseño experimental en bloques completamente randomizados (DBCR): cuatro repeticiones con seis concentraciones y empleándose 240 larvas por bioensayo. La Concentración Letal media $\left(\mathrm{CL}_{50}\right)$ en $\mu \mathrm{g}$ del metal $\mathrm{L}^{-1}$ a $48 \mathrm{~h}$ de exposición y el coeficiente de

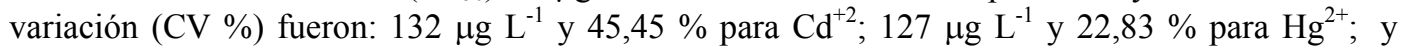
finalmente $18.730 \mu \mathrm{g} \mathrm{L}^{-1}$ y $33 \%$ para $\mathrm{Pb}^{2+}$. La variabilidad del ensayo con C. calligraphus es comparada con otros organismos biológicos dulceacuícolas al nivel mundial.

Palabras clave: Bioensayo, Chironomus, cadmio, ecotoxicología, macroinvertebrado, mercurio, plomo, repetibilidad.

\begin{abstract}
Environmental Risk Assessment (ERA) of freshwater environment in Peru needs the employment of standardized biological organisms such as the larval midge Chironomus calligraphus Goeldi. The biological organisms to be employed as ecotoxicological tools should be easy, practical, sensitive and reproductible. The aim of this research was to evaluate $\mathrm{Cd}^{+2}, \mathrm{Hg}^{+2}$ and $\mathrm{Pb}^{+2}$ as cadmium chlorure $\left(\mathrm{CdCl}_{2}\right)$, mercury chlorure $\left(\mathrm{HgCl}_{2}\right)$ and lead nitrate $\left(\mathrm{Pb}_{3}(\mathrm{NO})_{2}\right)$ forms, on the mortality of first instar larva of diptera C. calligraphus at $48 \mathrm{~h}$ exposure and in this way determinate its variability. The species was acclimated and reared under standardized laboratory conditions. Six bioassays of acute static toxicity for each metal were done, in each one a randomized block experimental design (RBED) was used with four repetitions of five concentrations and employing 240 larvae for each bioassay. The median lethal concentration $\left(\mathrm{LC}_{50}\right)$ in $\mu \mathrm{g}$ metal $\mathrm{L}^{-1}$ at $48 \mathrm{~h}$ exposure and the coefficient of variation $(\mathrm{CV} \%)$ were: $132 \mu \mathrm{g} \mathrm{L}^{-1}$ and 45,45 $\%$ for $\mathrm{Cd}^{+2} ; 127 \mu \mathrm{g} \mathrm{L}^{-1}$ and $22,83 \%$ for $\mathrm{Hg}^{2+}$; and finally $18.730 \mu \mathrm{g} \mathrm{L}^{-1}$ and $33 \%$ for $\mathrm{Pb}^{2+}$. The variability of the essay of $C$. calligraphus is compared with other freshwater biological organisms all over the world.
\end{abstract}

Key words: Bioassay, Chironomus, cadmium, ecotoxicology, lead, macroinvertebrate, mercury, repeatability.

\section{Introducción}

La ecotoxicología es la ciencia que describe y predice el comportamiento de las sustancias en el medio ambiente y las respuestas biológicas del sistema para así, finalmente evaluar el riesgo asociado con estas emisiones (Delvalls \& Conradi, 2000). Los bioensayos ecotoxicológicos en el laboratorio con un determinado organismo biológico y el uso de controles permiten predecir el efecto de las sustancias químicas tóxicas (Calow, 1993). Los organismos biológicos para ser usados como herramientas ecotoxicológicas requieren ser sencillos, prácticos, sensibles y repetibles (Iannacone et al., 1998; Alayo \& Iannacone, 2002). La APHA (1989) señala como protocolos estandarizados para la realización de bioensayos de toxicidad aguda y crónica acuática a las larvas de los insectos quironómidos, principalmente Chironomus tentans Fabricius, Chironomus riparius Meigen y Chironomus plumosus (Linnaeus) (Mereggalli et al., 2002; Lahr et al., 2003; Martinez et al., 2003). Estos mosquitos quironómidos son abundantes en los ambientes dulceacuícolas y

1 Laboratorio de Ecofisiología Animal. Facultad de Ciencias Naturales. Universidad Nacional Federico Villarreal (FCCNM-UNFV). Calle San Marcos 383, Lima 21, Perú.

Correo electrónico: joseiannacone@hotmail.com / joselorena@terra.com. 
Diciembre 2003

componente importante en las comunidades acuáticas detritívoras (Iannacone et al., 2000; Crane et al., 2002; Sánchez \& Tarazona, 2002).

Chironomus calligraphus Goeldi, es un insecto Pan-Americano, de distribución predominantemente Neotropical, y que también ocurre en la región Neártica Sur, muy común en los ambientes acuáticos epicontinentales de la ciudad de Lima, Perú (Iannacone et al., 1999, 2000; Spies et al., 2002). C. calligraphus ha demostrado una alta sensibilidad a metales pesados (Iannacone et al., 1998; Iannacone \& Dale, 1999; Arrascue et al., 2001) y a plaguicidas (Iannacone \& Alvariño, 1998, 2000). Para la evaluación del riesgo ambiental (ERA) de los ambientes acuáticos epicontinentales en el Perú se requiere del empleo de organismos biológicos estandarizados como la larva del insecto $C$. calligraphus.

Los metales pesados, como el cadmio, mercurio y plomo, producen efectos deletéreos en las aguas naturales (Weber, 1996; Iannacone \& Alvariño, 1999; Fernández \& Beiras, 2001).

En Latinoamérica existen algunas experiencias de ejercicios de intra e intercalibración empleando baterías de bioensayos, que incluyen artrópodos, para la evaluación de tóxicos de referencias con el fin de determinar el grado de repetibilidad, precisión o variabilidad de estas pruebas ecotoxicológicas (Forget et al., 2000). Así se han realizado en países como Argentina (Ronco et al., 2000), Chile (Castillo \& Schäfer, 2000; Castillo et al., 2000a), Colombia (Díaz-Baez \& Perez, 2000), Costa Rica (Castillo et al,. 2000b) y México (Pica et al., 2000). En el Perú, aún no se tienen este tipo de experiencias para los bioensayos ecotoxicológicos.

Por lo que, el objetivo de este trabajo fue evaluar el grado de variabilidad o repetibilidad del bioensayo que emplea a $C$. calligraphus usando al cadmio, mercurio y plomo como tóxicos de referencia.

\section{Materiales y métodos \\ Chironomus calligraphus}

La colección, identificación y cría estandarizada de C. calligraphus, así como el protocolo de bioensayo siguió el procedimiento detallado descrito por Iannacone et al. (2002a). Los ensayos ecotoxicológicos con $C$. calligraphus se iniciaron con larvas de primer estadio dentro de $24 \mathrm{~h}$ de haber eclosionado de las masas de huevos procedente de la Planta de Tratamiento de Aguas residuales domésticas de Villa El Salvador, Lima, Perú durante el 2000 al 2001. Los ejemplares se separaron al azar de los frascos de eclosión y recuperación de las larvas de primer estadio. Diez larvas de primer estadio se distribuyeron al azar en cada concentración de las muestras con metales evaluadas en cada una de las cuatro repeticiones del ensayo experimental. Cada repetición incluyó cinco concentraciones ensayadas y el control. Las larvas se consideraron muertas si no fueron capaces de moverse coordinada y normalmente cuando fueron pinchadas ligeramente con un alfiler luego de $48 \mathrm{~h}$ de exposición. Se emplearon un total de 240 larvas por ensayo. Los bioensayos fueron repetidos seis veces secuencialmente. $\mathrm{El} \mathrm{pH}$ se midió mediante un potenciómetro. $\mathrm{El} \mathrm{pH}$ se midió en dos réplicas al inicio del ensayo y a las $48 \mathrm{~h}$ de exposición. La temperatura se mantuvo regulada a $20{ }^{\circ} \mathrm{C} \pm 3{ }^{\circ} \mathrm{C}$.

\section{Metales y Físico-química del agua}

Las soluciones madre para los iones tóxicos de cadmio, mercurio y plomo fueron preparadas de la sal metálica a base del cloruro de cadmio $\left(\mathrm{CdCl}_{2}\right)$, cloruro de mercurio $\left(\mathrm{HgCl}_{2}\right)$ y nitrato de plomo $\left(\mathrm{Pb}_{3}(\mathrm{NO})_{2}\right)$ a una concentración de $100 \mathrm{mg} \mathrm{L}^{-1}$. Todas las concentraciones se registraron en concentraciones nominales de ión de metálico. Los matraces aforados previamente fueron lavados con ácido nítrico al $10 \% \mathrm{y}$ luego enjuagados en agua destilada y mantenidos a $4{ }^{\circ} \mathrm{C}$ (Iannacone et al., 2000). El pH fue ajustado al inicio del ensayo a 7 con una solución $\mathrm{NaOH} 0,1 \mathrm{M}$ o con $\mathrm{H}_{2} \mathrm{SO}_{4}$ 0,1M. Los datos de oxígeno disuelto se midieron mediante el método de Winkler (APHA, 1989). En el ensayo con $\mathrm{Cd}^{2+}$ se emplearon las siguientes cinco concentraciones $125 \mu \mathrm{g} \mathrm{L}^{-1}, 250 \mu \mathrm{g} \mathrm{L}^{-1}, 500 \mu \mathrm{g} \mathrm{L}^{-1}$, $1.000 \mu \mathrm{g} \mathrm{L}^{-1}$ y $2.000 \mu \mathrm{g} \mathrm{L}{ }^{-1}$. En el ensayo con $\mathrm{Hg}^{2+}$ se emplearon las siguientes cinco concentraciones 62,5 $\mu \mathrm{g} \mathrm{L}^{-1}, 125 \mu \mathrm{g} \mathrm{L}^{-1}, 250 \mu \mathrm{g} \mathrm{L}^{-1}, 500 \mu \mathrm{g} \mathrm{L}^{-1}$ y $1.000 \mu \mathrm{g}$ $\mathrm{L}^{-1}$. En el ensayo con $\mathrm{Pb}^{2+}$ se emplearon las siguientes cinco concentraciones $6.250 \mu \mathrm{g} \mathrm{L}^{-1}, 12.500 \mu \mathrm{g} \mathrm{L}^{-1}$, $25.000 \mu \mathrm{g} \mathrm{L}^{-1}, 50.000 \mu \mathrm{g} \mathrm{L}^{-1}$ y $100.000 \mu \mathrm{g} \mathrm{L}{ }^{-1}$. Para el $\mathrm{Cd}^{2+}, \mathrm{Hg}^{2+}, \mathrm{Pb}^{2+}$ las cinco concentraciones siguieron un factor de incremento de 2. Las principales características fisicoquímicas del agua destilada son las indicadas por Iannacone et al. (2002b).

Diseño Experimental y Análisis de datos

Se determinaron la Concentración Letal media $\left(\mathrm{CL}_{50}\right)$ promedio, su desviación estándar y su Coeficiente de Variación (CV \%) para los bioensayos de $C$. calligraphus expuestos al cadmio, mercurio y plomo utilizando los resultados de las sumas de las cuatro repeticiones de cada concentración. Las diferencias entre las concentraciones se evaluaron a través de un Análisis de Varianza (ANDEVA), previa transformación de los datos a raíz cuadrada del arcoseno, con el fin de ajustar los datos a la distribución normal (Zar, 1996). Se utilizaron los bioensayos como bloques y las variables respuestas provinieron de las sumas de las cuatro repeticiones de cada concentración de cada ensayo. En el caso de existir diferencias significativas entre las concentraciones se realizó una Prueba de Significación DVS (Diferencia Verdaderamente Significativa) de Tukey (Daniel, 1993).

Las $\mathrm{CL}_{50} \mathrm{~S}$ se calcularon usando un programa computarizado de la EPA versión 1,5 -1993 (USEPA, 
1993). Estos valores fueron obtenidos a partir del análisis de regresión lineal entre el logaritmo de la concentración de las muestras con metales y la mortalidad Probit (porcentaje transformado). Estos modelos de regresión lineal fueron verificados usando el estadístico $\chi^{2}$ (Martín \& Holdich, 1986). Se calcularon los valores de NOEC (Concentración de efectos no observables) y LOEC (Concentración más baja de efectos observables) para cada metal.

\section{Resultados}

La secuencia en orden de toxicidad de $C$. calligraphus expuesto a los tres metales fue: $\mathrm{Hg}^{2+}>$ $\mathrm{Cd}^{2+}>\mathrm{Pb}^{2+}$ (Tabla 1).

Las Tablas 2, 3 y 4 señalan los porcentajes de mortalidad para cada una de las cinco concentraciones y el control en cada una de los seis bioensayos para el cadmio, mercurio y plomo, respectivamente. Para todos los casos el CV (\%) fue menor del $50 \%$, a excepción del control del ensayo con cadmio que fue del $75,42 \%$. Sin embargo, en ninguno de los casos la mortalidad en el control sobrepasó el $20 \%$. La prueba de Tukey mostró que el LOEC para el $\mathrm{Cd}^{2+}, \mathrm{Hg}^{2+}$ y $\mathrm{Pb}^{2+}$ fue de $62,5,125$ y 6.250 ug L$^{-1}$, respectivamente. En cambio, el NOEC para el $\mathrm{Cd}^{2+}, \mathrm{Hg}^{2+}$ y $\mathrm{Pb}^{2+}$ fue de $<62,5<125 \mathrm{y}<6.250 \mathrm{ug} \mathrm{L}^{-1}$, respectivamente.

Para C. calligraphus, el valor promedio de la $\mathrm{CL}_{50}$ al cadmio de $132 \mu \mathrm{g} \mathrm{L}^{-1}$ ocupa la posición tercera en comparación a otras diez especies del género Chironomus (Tabla 5).

Para C. calligraphus, el valor promedio de la $\mathrm{CL}_{50}$ al mercurio de $130 \mu \mathrm{g} \mathrm{L}^{-1}$ ocupa la posición cuarta en comparación a otras siete especies del género Chironomus (Tabla 6). Para C. calligraphus, el valor promedio de la $\mathrm{CL}_{50}$ al plomo de $18.732 \mu \mathrm{g} \mathrm{L}^{-1}$ ocupa la posición primera en comparación a otras siete especies del género Chironomus (Tabla 7). La Tabla 8 , señala que $C$. calligraphus ocupa la posición $12^{\text {va }}$ $\left(\right.$ para el $\left.\mathrm{Hg}^{2+}\right), 15^{\text {va }}$ (para el $\mathrm{Pb}^{2+}$ ) y $18^{\text {va }}$ (para el $\mathrm{Cd}^{2+}$ ) entre 29 bioensayos ecotoxicológicos empleando diferentes componentes de la biota animal.

\section{Discusión}

Las especies propuestas como herramientas para bioensayos ecotoxicológicos, requieren como requisitos para uso no solo que sean sensibles, sino que los ensayos sean reproducibles con poca variabilidad en su respuesta (Magdaleno et al., 1997; Steevens \& Benson, 1998). C. calligraphus es una especie con alta sensibilidad a metales pesados, plaguicidas y a muestras ambientales (Iannacone et al., 2000; Arrascue et al, 2001). Sus valores de $\mathrm{CL}_{50}$ para el cadmio, mercurio y plomo tienen en general el mismo orden de magnitud que otras especies del género (Tablas 5, 6 y 7). En un estudio previo, Iannacone \& Dale (1999) encontraron una sensibilidad de C. calligraphus al mercurio (seis veces mayor); al plomo (cinco veces menor) y al mercurio (dos veces menor que la encontrada en el presente estudio). Sin embargo estos resultados correspondieron para cada metal a un bioensayo sin repeticiones.

Entre los numerosos factores bióticos que pudieran influir en la toxicidad del cadmio, mercurio y plomo sobre las especies de la familia Chironomidae, tenemos a la especie, estado de desarrollo y edad. Los valores comparativos de $\mathrm{CL}_{50}$ expuestos al cadmio, mercurio y plomo para las diferentes especies del Chironomus señalados en la Tablas 5,6 y 7 señalan diferencias por ser especies diferentes, así como estados de desarrollo y edades diferentes por ser utilizados formas larvarias del primer al cuarto estadio. En general se considera que las formas larvarias de estadios tempranos son más sensibles que los estadios superiores (Tablas 5, 6 y 7). Las larvas de primer estadio (plantónicas) son más sensibles que las de segundo estadio (bentónicas) de C. riparius y de las de cuarto estadio (bentónicas) de C. decorus. Además, entre los factores abióticos se señala al tiempo de exposición como un factor de importancia que influiría en la ecotoxicidad de los tres metales pesados (Peck et al., 2002).

Los coeficientes de variación (CV \%) obtenidos para los 29 ensayos empleando especies biológicas acuáticas pertenecientes a diferentes taxas, variaron entre 2,1 a más de $100 \%$ (Tabla 8). El ensayo con $C$. calligraphus ocupa la posición $12^{\mathrm{va}}, 15^{\mathrm{va}}$ y $18^{\mathrm{va}}$ para $\mathrm{Hg}^{2+}, \mathrm{Pb}^{2+}$ y $\mathrm{Cd}^{2+}$, respectivamente, el cual fue aceptable al ser menor al $50 \%$. Uno de los factores que estaría influyendo en la variabilidad encontrada en el ensayo estático de mortalidad con C. calligraphus expuestos a los tres metales a $48 \mathrm{~h}$ de exposición, es la estación del año y la calidad del agua (contenido de oxígeno, $\mathrm{pH}$, sólidos suspendidos, etc.) del lugar colecta de los organismos, ya que los quironómidos en la fase de huevos son obtenidos de cuerpos de agua artificiales en forma periódica a través de las diferentes estaciones del año y luego criados artificial y parcialmente en condiciones estandarizadas de laboratorio (Milani et al., 2003). Las especies criadas artificialmente en forma continua durante todo su ciclo vital tienden a presentar menor variabilidad en su respuesta que las especies criadas parcialmente y obtenidas en forma periódica del campo (APHA, 1989; Calow, 1993).

\section{Agradecimientos}

Al Laboratorio de Ecofisiología Animal de la Universidad Nacional Federico Villarreal, Lima, Perú.

\section{Literatura citada}

Alayo M. \& Iannacone J. 2002. Ensayos ecotoxicológicos con petroleo crudo, Diesel 2 y Diesel 6 con dos subespecies de Brachionus plicatilis Müller 1786 (Rotifera: Monogononta). Gayana. 66: 45-58. 
APHA (AMERICAN PUBLIC HEALTH ASSOCIATION), AWWA (AMERICAN WATER WORKS ASSOCIATION), WPCF (WATER POLLUTION CONTROL FEDERATION). 1989. Standard methods for examination of water and wastewater. $17^{\text {th }}$. Ed. American Health Association. Washington, D.C.

Arrascue A., Iannacone J., Alvariño L., Basilio S. \& Lazcano C. 2001. El insecto Chironomus calligraphus Goeldi y la bacteria Escherichia coli como ensayos ecotoxicológicos para evaluar sedimentos elutriados dulceacuícolas. Rev. per. Ent. 42: 159-173.

Calow P. 1993. Handbook of ecotoxicology. 478 p. Vol. I. Blackwell Science, Sheffield, UK.

Castillo G.C. \& Schäfer L. 2000. Evaluation of a bioassay battery for water toxicity testing: A Chilean experience. Environm. Toxicol. 15: 331337.

Castillo G.C., Vila I.C. \& Neild E. 2000a. Ecotoxicity assessment of metals and wastewater using multitrophic assays. Environm. Toxicol. 15: 370375.

Castillo L.E., Pinnock N. \& Martinez E. 2000b. Evaluation of a battery of toxicity tests for use in the assessment of water quality in a Costa Rican laboratory. Environm. Toxicol. 15: 312-321.

Crane M., Sildanchandra W., Kheir R. \& Callaghan R. 2002. Relationships between biomarker activity and development endpoints in Chironomus riparius Meigen exposed to an organophosphate insecticide. Ecotoxicol. Environm. Saf. 53: 361369.

Daniel W.W. 1993. Bioestadística. Tercera Edición. Editorial Limusa (México).

Delvalls T.A. \& Conradi M. 2000. Advances in marine ecotoxicology: laboratory tests versus field assessment data on sediment quality studies. Ciencias Marinas. 26: 39-64.

Díaz-Baez M.C. \& Perez J.B. 2000. Intralaboratory experience with a battery of bioassays: Colombia experience. Environm. Toxicol.15: 297-303.

Espiritu E.Q., Janssen C.R. \& Persoone G. 1995. Cyst-based toxicity tests. VII. Evaluation of the 1h enzymatic inhibition test (Fluotox) with Artemia nauplii. Environm. Toxicol. Water Qual. 10: 2534.

Fargasova A. 2001. Winter third- to fourth- instar larvae of Chironomus plumosus as bioassay tools for assessment of acute toxicity of metals and their binary combinations. Ecotoxicol. Environm. Saf. 48: $1-5$.

Fernández N. \& Beiras N. 2001. Combined toxicity of dissolved mercury with Copper, lead and cadmium on embryogenesis and early larval growth of the Paracentrotus lividus sea-urchin. Ecotoxicol. 10: 263-271.
Forget G., Sánchez A., Arkhipchuk V., Beauregard T., Blaise C., Castillo G., Castillo L.E., Diaz-Baez M.C., Pica Y.G., Ronco A., Srivastava R.C. \& Dutka B.J. 2000. Preliminary data of a singleblind, multicountry trial of six bioassays for water toxicity monitoring. Environm. Toxicol. 15: 362369.

Greene J.C., Miller W.E., De Bacon M.K., Long M. A. \& Bartel S. 1985. A comparison of three microbial assay procedures for measuring toxicity of chemical residues. Arch. Environm. Contam. Toxicol. 14: 659-667.

Iannacone J., Dale W. \& Alvariño L. 2000. Monitoreo ecotoxicológico del río Rímac (Lima-Perú) empleando a Chironomus calligraphus Goeldi (Diptera: Chironomidae). Rev. Chil. Ent. 27: 2534.

Iannacone J., Alvariño L. \& Gutierrez A. 1999. Cinco ensayos ecotoxicológicos para evaluar metales pesados en el agua dulce. Bol. Soc. Quím. Perú 65: 30-45.

Iannacone J. \& Alvariño L. 1998. Ecotoxicidad aguda del insecticida organofosforado temephos sobre Chironomus calligraphus Goeldi (Diptera: chironomidae). Acta Ent. Chilena. 22: 51-53.

Iannacone J. \& Alvariño L. 1999. Acute ecotoxicity of heavy metals using juveniles of freshwater snail Physa venustula (Gould, 1847) (Mollusca). Gayana. 63: 101-110.

Iannacone J. \& Alvariño L. 2000. Chironomus calligraphus Goeldi y Moina macrocopa (Sars) como herramientas ecotoxicológicas para la evaluación del lindano y clorpirifos. Bol. Soc. Biol. Concepción (Chile). 71: 33-39.

Iannacone J. \& Dale W.E. 1999. Protocolo de bioensayo ecotoxicológico para evaluar metales pesados contaminantes de agua dulce con Chironomus calligraphus (Diptera: Chironomidae) y el Crustáceo Moina macrocopa (Crustácea: Cladócera), en el río rímac. Rev. per. Ent. 41: 111120.

Iannacone J., Alvariño L. \& Dale W.E. 1998. Pruebas ecotoxicológicas como una herramienta para la evaluación del impacto ambiental de los ecosistemas acuáticos. Bol. Lima (Perú). 113: 5368.

Iannacone J., Alvariño L. \& Mansilla J. 2002a. Actividad insecticida de cuatro extractos botánicos sobre larvas de los mosquitos Culex quinquefasciatus (Diptera: Culicidae) y Chironomus calligraphus (Diptera: Chironomidae). Wiñay Yachay. 6: 59-74.

Iannacone J., Caballero C. \& Alvariño L. 2002b. Employing the freshwater snail Physa venustula Gould as an ecotoxicological tool for environmental risk assessment by pesticides. Agri. Téc. (Chile). 62: 212-225. 
Janssen C. 1998. Alternative assay for routine toxicity assessments. A review. Chapter 26. pp. 813-839. In: Ecotoxicology. G. Schüürmann, Markert B. Eds.

Khangarot B.S. \& Ray P.K. 1989. Sensitivity of midge larvae of Chironomus tentans Fabricius (Diptera Chironomidae) to heavy metals. Bull. Environm. Contam. Toxicol. 42: 325-330.

Lahr J., Maas-Diepeveen J.L., Stuijfzand S.C., Leonards P.E., Druke J.M., Lucker S., Espeldoorn A., Kerkum L.C., van Stee L.L. \& Hemdriks A.J. 2003. Response in sediment bioassays used in the Netherlands: can observed toxicity be explained by routinely priority pollutants?. Water Res. 37: 1691-1710.

Magdaleno A., Gomez C.E., Velez C.G. \& Accorinti J. 1997. Preliminary toxicity test using the green alga Ankistrodesmus falcatus. Environm. Toxicol. Water Qual. 12: 11-14.

Martin T.R. \& Holdich D.M. 1986. The acute lethal toxicity of heavy metals to peracarid crustaceans (with particular reference to freshwater asellids and gammarids). Water Res. 20: 1137-1147.

Martinez E.A., Moore B.C., Schaumloffel J. \& Dasgupta N. 2003. Morphological abnormalities in Chironomus tentans exposed to cadmium and copper spiked sediments. Ecotoxicol. Environm. Saf. 55: 204-212.

Mereggalli G., Bettinetti R., Pluymers L., Vermeulen A.C., Rossaro B. \& Ollevier F. 2002. Mouthpart deformities and nucleolus activity in fieldcollected Chironomus riparius larvae. Arch. Environm. Contam. Toxicol. 42: 405-409.

Milani D., Reynoldson T.B., Borgmann U. \& Kolasa J. 2003. The relative sensitivity of four benthic invertebrates to metals in spiked-sediment exposures and application to contaminated field sediment. Environ. Toxicol. Chem. 22: 845-854.

Postma J.F., Buckert de Jong M.C., Staats N. \& Davids C. 1994. Chronic toxicity of cadmium to Chironomus riparius (Diptera: Chironomidae) at different food levels. Arch. Envrion. Contam. Toxicol. 26: 143-148.

Peck M.R., Klessa D.A. \& Baird D.J. 2002. A tropical sediment toxicity test using the dipteran Chironomus crassiforceps to test metal bioavailability with sediment $\mathrm{pH}$ change in tropical acid-sulfate sediments. Environm. Toxicol. Chem. 21: 720-728.

Persoone G. 1996. Development and first validation of a "culture free" algal microbiotest: the algaltoxkit. In: Microscale toxicology, advances, techniques and practice, Wells, P.G., Lee K., Blaise, C. (eds.). CRC Publishers.

Pica G.Y., Trujillo G.D. \& Hernández H.S. 2000. Bioassay standardization for water quality monitoring in Mexico. Environm. Toxicol. 15: 322-330.
Qureshi S.A., Sakesena A.B. \& Singh V.P. 1980. Acute toxicity of four heavy metals to benthic fish food organims for the River Khan Ujjain. Int. J. Environm. Stu. $15: 59-61$.

Rao D.S. \& Saxena A.B. 1981. Acute toxicity of mercury, zinc, lead, cadmium and manganese to Chironomus sp. Int. J. Environ. Stud. 16 : 226-226.

Reish D.J. 1993. Effect of metals and organic compounds on survival and bioaccumulation in two species of marine gammaridean amphipod, together with a summary of toxicological research on this group. Jour. Nat. History. 27: 781-794.

Ronco A., Sobrero C., Grassi V., Kaminski L., Massolo L. \& Mina L. 2000. WaterTox bioassay intercalibration network: results from Argentina. Environm. Toxicol. 15: 287-296.

Rossino G.B. \& Ronco A.E. 1996. Acute toxicity bioassay using Daphnia obtusa as a test organism. Environm. Toxicol.Water Qual. 11: 255-258.

Sánchez P. \& Tarazona J.V. 2002. Development of a multispecies system for testing reproductive effects on aquatic invertebrates. Experience with Daphnia magna, Chironomus prasinus and Lymnaea peregra. Aquatic Toxicol. 60: 249-256.

Snell T.W., Moffat B.D., Janssen C. \& Persoone G. 1991. Acute toxicity tests using rotifers: III. Effects of temperature, strain and exposure time on the sensitivity of Brachionus plicatilis. Environm. Toxicol. Water Qual. 6: 63-75.

Spies M., Sublette J.E., Sublette M.F., Wülker W.F., Martin J., Hille A., Miller M.A. \& Witt K. 2002. Pan-american Chironomus calligraphus Goeldi, 1905 (Diptera: Chironomidae): Species or Complex?. Evidence from external morphology, karyology and DNA sequencing. Aquat. Insects. 24: 91-113.

Steevens J.A. \& Benson W.H. 1998. Hyalella azteca 10-day sediment toxicity test: Comparison of growth measurement endpoints. Environm. Toxicol. Water Qual. 13: 243-248.

USEPA. 1993. Probit analysis program. Version 1,5. U.S. Environmental Protection Agency. Washington D.C. U.S.A.

Watts M.M. \& Pascoe D. 2000. A comparative study of Chironomus riparius Meigen and Chironomus tentans Fabricius (Diptera: Chironomidae) in aquatic toxicity tests. Arch. Environm. Toxicol. 39: 299-306.

Weber D.N. 1996. Lead-induced metabolic imbalances and feeding alterations in juvenile fathead minnows (Pimephales promelas). Environm. Toxicol. Water Qual. 11: 45-51.

Willemsen A., Vaal M.A. \& De Zwart D. 1995. Microbiotests as tools for environmental monitoring. National Institute of Public Health and Environmental Planning (RIVM). The Netherlands, report No 9. 607042005. 
Diciembre 2003

Zar J.H. 1996. Bioestatistical Analysis. $3^{\text {th }}$ Ed. Prentice -Hall. Inc. Upper Saddle River, Jew Jersey.

Tablas citadas en el texto

Tabla 1. Valores de la $\mathrm{CL}_{50}$ y sus límites de confianza para los bioensayos realizados con los tres metales pesados

\begin{tabular}{llllllllll}
\hline & $\mathrm{Hg}^{2+}$ & \multicolumn{1}{c}{$\mathrm{Cd}^{2+}$} & \multicolumn{7}{c}{$\mathrm{Pb}^{2+}$} \\
\hline Repetición & L. Inf. & $\mathrm{CL}_{50}$ & L. Sup. & L. Inf. & $\mathrm{CL}_{50}$ & L. Sup. & L. Inf. & $\mathrm{CL}_{50}$ & L. Sup. \\
1 & 71 & 113 & 154 & 152 & 210 & 264 & 11.490 & 19.515 & 27.782 \\
2 & 45 & 83 & 121 & 138 & 179 & 217 & $*$ & 19.474 & $*$ \\
3 & 100 & 150 & 200 & 150 & 193 & 232 & 15.460 & 22.329 & 29.062 \\
4 & 102 & 152 & 198 & 37 & 86 & 120 & 19.620 & 27.593 & 34.974 \\
5 & 86 & 125 & 162 & 15 & 67 & 104 & 9.219 & 12.748 & 16.257 \\
6 & 110 & 154 & 197 & 11 & 59 & 99 & 7.485 & 10.735 & 13.915 \\
Promedio & 86 & 130 & 172 & 84 & 132 & 173 & 12.660 & 18.732 & 24.398 \\
D.E & 24 & 28 & 32 & 70 & 69 & 73 & 4.906 & 6.203 & 8.961 \\
\hline
\end{tabular}

L. inf. $=$ Límite inferior; $\mathrm{CL}_{50}=$ Concentración Letal media; $\mathrm{L}$. sup. $=$ Límite superior;

D.E. $=$ Desviación estandar; $(*)=$ No determinado.

Tabla 2. Porcentaje de Mortalidad de C. calligraphus expuestas al cadmio a $48 \mathrm{~h}$

\begin{tabular}{llllllllll}
\multicolumn{8}{c}{ Repeticiones (\% mortalidad) } \\
\hline$\mu \mathrm{g} \mathrm{L}{ }^{-1}$ & 1 & 2 & 3 & 4 & 5 & 6 & Promedio \pm DESig. & CV \\
\hline control & 15 & 10 & 10 & 2,5 & 2,5 & 2,5 & $7,08 \pm 5,34$ & a & 75,42 \\
125 & 32,5 & 32,5 & 32,5 & 67,5 & 75 & 77,5 & $52,92 \pm 22,61$ & $\mathrm{~b}$ & 42,72 \\
250 & 65 & 77,5 & 67,5 & 92,5 & 97,5 & 92,5 & $82,08 \pm 14$ & $\mathrm{c}$ & 17,06 \\
500 & 95 & 95 & 97,5 & 97,5 & 97,5 & 97,5 & $96,67 \pm 1,29$ & $\mathrm{~d}$ & 1,34 \\
1.000 & 97,5 & 100 & 100 & 100 & 100 & 100 & $99,58 \pm 1,02$ & $\mathrm{~d}$ & 1,02 \\
2.000 & 100 & 100 & 100 & 100 & 100 & 100 & $100 \pm 0$ & $\mathrm{~d}$ & 0 \\
\hline
\end{tabular}

$\mathrm{DE}=$ Desviación Estándar; $\mathrm{CV}=$ Coeficiente de Variación; Sig. $=$ Significancia.

Tabla 3. Porcentaje de mortalidad de C. calligraphus expuestas al mercurio a $48 \mathrm{~h}$. Repeticiones (\% mortalidad)

\begin{tabular}{llllllllll}
\hline $\mathrm{ug} / \mathrm{L}$ & 1 & 2 & 3 & 4 & 5 & 6 & \multicolumn{2}{c}{ Promedio \pm DESig.CV } \\
\hline control & 15 & 12,5 & 15 & 17,5 & 15 & $12,514,58 \pm 1,88$ & $\mathrm{a}$ & 12,90 \\
62,5 & 35 & 47,5 & 32,5 & 30 & 35 & 25 & $34,17 \pm 7,53$ & $\mathrm{~b}$ & 22,03 \\
125 & 70 & 67,5 & 52,5 & 52,5 & 50 & 45 & $56,25 \pm 10,09$ & $\mathrm{c}$ & 17,94 \\
250 & 72,5 & 77,5 & 67,5 & 72,5 & 87,5 & 75 & $75,42 \pm 6,79$ & $\mathrm{~d}$ & 9 \\
500 & 92,5 & 87,5 & 95 & 97,5 & 95 & 95 & $93,75 \pm 3,45$ & $\mathrm{e}$ & 3,68 \\
1.000 & 100 & 100 & 100 & 100 & 100 & $100100 \pm 0$ & $\mathrm{f}$ & 0 \\
\hline
\end{tabular}

$\overline{\mathrm{DE}}=$ Desviación Estándar; $\mathrm{CV}=$ Coeficiente de variación; Sig. $=$ Significancia.

Tabla 4. Porcentaje de mortalidad de C. calligraphus expuestas al plomo a $48 \mathrm{~h}$.

\begin{tabular}{llllllllll}
\hline \multicolumn{1}{l}{ Repeticiones (\% mortalidad) } \\
\hline ug L-1 & 1 & 2 & 3 & 4 & 5 & 6 & Promedio +/- DE & Sig. & CV \\
\hline Control & 17,5 & 20 & 10 & 12,5 & 10 & 10 & $13,33 \pm 4,38$ & a & 32,83 \\
6.250 & 37,5 & 42,5 & 25 & 20 & 27,5 & 35 & $31,25 \pm 8,48$ & ab & 27,13 \\
12.500 & 47.5 & 55 & 35 & 32,5 & 57,5 & 62,5 & $48,33 \pm 12,32$ & bc & 25,48 \\
25.000 & 65 & 62,5 & 57,5 & 47,5 & 77,5 & 82,5 & $65,42 \pm 12,89$ & $\mathrm{c}$ & 19,70 \\
50.000 & 75 & 67,5 & 80 & 77,5 & 95 & 95 & $81,67 \pm 11,14$ & $\mathrm{~d}$ & 13,64 \\
100.000 & 100 & 100 & 100 & 100 & 100 & 100 & $100 \pm 0$ & $\mathrm{e}$ & 0 \\
\hline
\end{tabular}

$\mathrm{DE}=$ Desviación estándar; $\mathrm{CV}=$ Coeficiente de variación; Sig. = Significancia. 
Tabla 5. Valores comparativos de $\mathrm{CL}_{50}$ con otras especies de Chironomus expuestas al cadmio.

\begin{tabular}{llll}
\hline Especie de Chironomus & $\begin{array}{l}\mathrm{CL}_{50} \\
\mu \mathrm{g} \mathrm{L}^{-1}\end{array}$ & $\begin{array}{l}\text { Tiempo de } \\
\text { Exposición (h) }\end{array}$ \\
\hline \hline Chironomus riparius Meigen & 13 & 48 & primer Milani et al. (2003) \\
Chironomus riparius Meigen & 16,2 & 72 & cuarto Postma et al. (1994) \\
Chironomus calligraphus Goeldi & 132 & 48 & primer Original \\
Chironomus calligraphus Goeldi & 280 & 48 & primer Iannacone \& Dale (1999) \\
Chironomus riparius Meigen & 1.200 & 240 & cuarto Watts \& Pascoe (2000) \\
Chironomus tentans Fabricius & 1.200 & 240 & cuarto Watts \& Pascoe (2000) \\
Chironomus calligraphus Goeldi & 3.670 & 24 & primer Iannacone \& Dale (1999) \\
Chironomus tentans Fabricius & 8.050 & 48 & tercero Khangarot \& Ray (1989) \\
Chironomus plumosus (Linnaeus) & 10.000 & 96 & tercero Fargasova (2001) \\
Chironomus tentans Fabricius & 23.250 & 24 & tercero Khangarot \& Ray (1989)
\end{tabular}

Tabla 6. Valores comparativos de $\mathrm{CL}_{50}$ con otras especies de Chironomus expuestas al mercurio.

\begin{tabular}{llll}
\hline Especie de Chironomus & $\mathrm{CL}_{50}$ & $\begin{array}{l}\text { Tiempo de } \\
\text { Exposición } \\
\text { (h) }\end{array}$ & Estadio Referencia \\
\hline \hline Chironomus calligraphus Goeldi & 20 & 48 & primer Iannacone \& Dale (1999) \\
Chironomus tentans Fabricius & 29 & 48 & tercero Khangarot \& Ray (1989) \\
Chironomus tentans Fabricius & 119 & 24 & tercero Khangarot \& Ray (1989) \\
Chironomus calligraphus Goeldi & 127 & 48 & primer Original \\
Chironomus calligraphus Goeldi & 130 & 24 & primer Iannacone \& Dale (1999) \\
Chironomus tendipes & 664 & 48 & tercero Rao \& Saxena (1981) \\
Chironomus sp. & 1.800 & 40 & tercero Qureshi et al. (1980) \\
& & &
\end{tabular}

Tabla 7. Valores comparativos de $\mathrm{CL}_{50}$ con otras especies de Chironomus expuestas al plomo.

\begin{tabular}{llll} 
Especie de Chironomus & $\begin{array}{l}\mathrm{CL}_{50} \\
\mu \mathrm{g} \mathrm{L}^{-1}\end{array}$ & $\begin{array}{l}\text { Tiempo de } \\
\text { Exposición (h) }\end{array}$ & Estadio Referencia \\
\hline \hline Chironomus calligraphus Goeldi & 18.730 & 48 & primer Original \\
Chironomus tentans Fabricius & 34.670 & 48 & tercero Khangarot \& Ray (1989) \\
Chironomus tendipes & 50.000 & 48 & tercero Rao \& Saxena (1981) \\
Chironomus tentans Fabricius & 52.870 & 24 & tercero Khangarot \& Ray (1989) \\
Chironomus calligraphus Goeldi & 77.180 & 48 & primer Iannacone \& Dale (1999) \\
Chironomus calligraphus Goeldi & 13.030 & 24 & primer Iannacone \& Dale (1999) \\
Chironomus sp. & 200.000 & 40 & tercero Qureshi et al. (1980)
\end{tabular}


ENSAYO ECOTOXICOLÓGICO CON Chironomus calligraphus PARA EVALUAR CADMIO, MERCURIO Y PLOMO

Diciembre 2003

Tabla 8. Variabilidad o repetibilidad de los bioensayos empleando diferentes especies acuáticas.

\begin{tabular}{|c|c|c|c|}
\hline Taxa & Especie & $\mathrm{CV} \%$ & Referencia \\
\hline Pez & Onchorhynchus mykiss & 2,1 & Castillo et al. (2000) \\
\hline Microalga & Raphidocelis subcapitata & 8 & Persoone (1996) \\
\hline Echinodermatha & Paracentrotus lividus & 11 & Fernández \& Beiras (2001) \\
\hline Bacteria & Vibrio fisheri (Microtox) & 12 & Janssen (1998) \\
\hline Celenterado & Hydra attenuata & 13 & Diaz-Baez \& Perez (2000) \\
\hline Planta & Lactuca sativa & 14,9 & Forget et al. (2000) \\
\hline Rotifera & Brachionus plicatilis & 15 & Snell et al. (1991) \\
\hline Microcrustáceo & Artemia franciscana & 18 & Espiritu et al. (1995) \\
\hline Microalga & Raphidocelis subcapitata & 18 & Janssen (1998) \\
\hline Planta & Allium cepa & 20 & Forget et al. (2000) \\
\hline Microcrustáceo & Daphnia magna & 20 & Janssen (1998) \\
\hline Insecta & Chironomus calligraphus & 22,83 & Original $\left(\mathrm{Hg}^{2+}\right)$ \\
\hline Microcrustáceo & Daphnia magna & 26 & Willemsen et al. (1995) \\
\hline Microcrustáceo & Daphnia obtusa & 30 & Rossini \& Ronco (1996) \\
\hline Insecta & Chironomus calligraphus & 33 & Original $\left(\mathrm{Pb}^{2+}\right)$ \\
\hline Microalga & Ankistrodesmus falcatus & 40 & Magdaleno et al. (1997) \\
\hline Microcrustáceo & Hyalella azteca & 40 & Steevens \& Benson (1998) \\
\hline Insecta & Chironomus calligraphus & 45,45 & Original $\left(\mathrm{Cd}^{2+}\right)$ \\
\hline Planta & Lactuca sativa & 46 & Forget et al. (2000) \\
\hline Microcrustáceo & Artemia franciscana & 47 & Janssen (1998) \\
\hline Microcrustáceo & Corophium insidiosum & 56 & Reish ( 1993) \\
\hline Bacteria & Muta-Cromo Placa & 60 & Pica et al. (2000) \\
\hline Bacteria & Vibrio fisheri (Microtox) & 65 & Greene et al. (1985) \\
\hline Nemátoda & Panagrellus redivivus & 67 & Diaz-Baez \& Perez (2000) \\
\hline Microrganismo & Bacillus cereus & 70 & Forget et al. (2000) \\
\hline Microcrustáceo & Daphnia magna & 71 & Forget et al. (2000) \\
\hline Microcrustáceo & Elasmopus bampo & 72 & Reish ( 1993) \\
\hline Microcrustáceo & Moina macrocopa & 77 & Iannacone \& Dale (1999) \\
\hline Nemátoda & Panagrellus redivivus & $>100$ & Pica et al. (2000) \\
\hline
\end{tabular}

CV \% = Coeficiente de Variación 